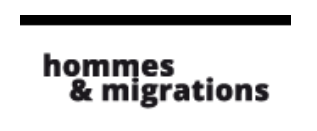

\section{Hommes \& migrations}

Revue française de référence sur les dynamiques

migratoires

$1303 \mid 2013$

Diasporas marocaines

\title{
Du couscous au foie gras
}

L'investissement des Marocains du monde dans le secteur de la gastronomie et ses répercussions dans le pays d'origine

Hicham Jamid et Marie Caquel

\section{(2) OpenEdition}

\section{Journals}

Édition électronique

URL : http://journals.openedition.org/hommesmigrations/2555

DOI : 10.4000/hommesmigrations.2555

ISSN : 2262-3353

Éditeur

Musée national de l'histoire de l'immigration

Édition imprimée

Date de publication : 1 juillet 2013

Pagination : 67-72

ISBN : 978-2-919040-23-0

ISSN : $1142-852 X$

Référence électronique

Hicham Jamid et Marie Caquel, « Du couscous au foie gras », Hommes \& migrations [En ligne], 1303 | 2013, mis en ligne le 31 décembre 2015, consulté le 01 mai 2019. URL : http://

journals.openedition.org/hommesmigrations/2555; DOI : 10.4000/hommesmigrations.2555 


\title{
DU COUSCOUS \\ AU FOIE GRAS \\ L'INVESTISSEMENT DES MAROCAINS DU MONDE DANS LE SECTEUR DE LA GASTRONOMIE ET SES RÉPERCUSSIONS DANS LE PAYS D'ORIGINE
}

par HICHAM JAMID, étudiant en master Migrations et développement durable, Ormes, université Ibn Zohr (Agadir), et MARIE CAQUEL, doctorante en histoire culturelle, université de Lorraine

\author{
Les saveurs venues d'ailleurs attirent les consommateurs \\ des deux côtés de la Méditerranée. Dans ce contexte \\ de mondialisation des pratiques culinaires, les entrepreneurs \\ migrants marocains cherchent à développer de nouveaux \\ marchés. En France, en complément de restaurants dédiés à leur \\ gastronomie nationale, ils ouvrent des commerces ethniques \\ proposant des produits de toutes origines. Au Maroc, \\ ils n'hésitent pas à renouveler l'offre culinaire afin de satisfaire \\ le besoin d'exotisme des couches sociales les plus aisées.
}

\section{Une réputation mondiale très positive}

La recherche interdisciplinaire sur les phénomènes migratoires ouvre actuellement de nouveaux champs qui mettent en relief les liens entre pays d'origine et pays d'installation en vue d'étudier les chemins de recomposition identitaire des migrants. Les investissements des Marocains du monde et la gastronomie forment un couple permettant de pénétrer ce phénomène.

L'histoire culturelle de l'alimentation fait, en effet, l'objet de publications novatrices légitimées par l'institution universitaire ${ }^{1}$. C'est ainsi que le colloque organisé à Bakou² en 2011 a montré la per- méabilité des frontières culturelles en matière de gastronomie.

La même année, un sondage de l'institut TNS Sofres ${ }^{3}$ indique que le couscous est le troisième plat préféré des Français, un symbole peut-être, mais ô combien révélateur des échanges culturels stimulés par les migrations. À travers l'étude ${ }^{4}$ des commerces et des restaurants marocains dans le pays d'installation puis de celle des investissements des migrants transnationaux dans ces mêmes secteurs au Maroc, l'enjeu est de soulever les questions d'identités multiples. 


\section{Une réputation mondiale au-delà des appartenances ethniques}

La cuisine marocaine dispose d'une réputation mondiale très positive diffusée notamment à travers le film d'Alfred Hitchcock, L'Homme qui en savait trop (1956). La scène dans le restaurant DarEssalam de Marrakech montre combien, pour un couple bourgeois (américain en l'occurrence), manger avec les mains n'est pas rebutant lorsqu'il s'agit de déguster un tajine dont le raffinement n'a rien à envier aux cuisines européennes et internationales. C'est pourquoi la clientèle non maghrébine représente une bonne partie du chiffre d'affaires pour les chefs marocains interrogés qui ont ouvert leur restaurant: "On a fait une étude de marché pour voir si ça pouvait fonctionner, les avantages du quartier, ce qui y manquait..." Néanmoins, ce restaurateur de Nancy regrette :

Si la population originaire du Maghreb, de Turquie et d'autres pays musulmans constitue un marché important en France de par

ses pratiques culturelles liées à l'islam et aux origines géographiques, elle a changé

dans sa structure et donc dans ses goûts "Nous sommes situés juste à côté du quartier Saint-Nicolas $^{5}$, où les gens ont l'habitude d'aller lorsqu'ils veulent manger un couscous. Il faudrait qu'ils fassent juste l'effort de quelques dizaines de mètres de plus pour venir le déguster chez nous!" Les habitudes et les représentations liées au quartier ont parfois la vie dure, c'est pourquoi ce chef cuisinier a misé sur 1"'authenticité", non sans une certaine prise de risque, pour attirer une clientèle qui trouvera des recettes et des saveurs inexistantes ailleurs. Chez lui, le couscous est cuit à la vapeur à trois reprises comme le veut la recette tradition- nelle et l'unique vin présent sur la carte ne contient pas d'alcool. Cependant, la majorité des autres restaurants marocains de notre étude, afin de capter une clientèle plus large et plus internationale, présente, d'une part, une carte plus "ouverte" à la cuisine du monde et, d'autre part, un large choix de vins et d'apéritifs. Salah, un autre restaurateur, nous confie: "Ma viande est halal, mais je mets des apéritifs et du vin sur ma carte, sinon mon restaurant ne pourrait pas marcher."

Néanmoins, la population européenne ne se contente pas d'aller au restaurant pour répondre à ses envies d'exotisme ${ }^{6}$, elle prétend également cuisiner "à la marocaine". Pour cela, elle se rend dans les commerces dits "ethniques". Ceux-ci sont, en effet, de plus en plus concernés par les phénomènes de mondialisation et d'évolution des pratiques qui ont un impact sur les stratégies et le développement commercial des investisseurs. Alors que Michèle Guillon et Isabelle Taboada-Leonetti ${ }^{8}$ affirmaient, en 1986, que le commerce ethnique était "pratiqué par certains groupes ethniques en direction de leur propre communauté $e^{-1}$, on ne peut plus le certifier aujourd'hui tant la clientèle de ces magasins d'alimentation a évolué.

Si la population originaire du Maghreb, de Turquie et d'autres pays musulmans constitue un marché important en France de par ses pratiques culturelles liées à l'islam et aux origines géographiques, elle a changé dans sa structure et donc dans ses goûts. Les enfants d'immigrés, tout en gardant dans leur mode de consommation certaines pratiques leur venant de leurs parents ${ }^{10}$ et notamment celles concernant la viande halal ${ }^{11}$, aspirent à manger comme leurs amis ou collègues. Ainsi, dans les épiceries, aux côtés des traditionnels sacs de couscous, épices, lait fermenté, tajines, gasâa ${ }^{12}$ et théières, on trouve des boissons gazeuses "arabes"

5. Quartier d'arrivée et d'implantation de migrants depuis les années 1950, situé dans le centre historique de Nancy. 6. Anaïs Fléchet, “L'exotisme comme objet d'histoire”, in Hypothèses, n 1, 2007, pp. 15-26. 7. Emmanuel Ma Mung, "L'expansion du commerce ethnique : Asiatiques et Maghrébins dans la région parisienne", in Revue européenne des migrations internationales, vol. 8, n¹, 1992, pp. 39-59. 8. Dans Michèle Guillon, Isabelle Taboada-Leonetti, Le Triangle de Choisy. Un quartier chinois à Paris, Paris, Cieme/L'Harmattan, 1986. 9. Citées par Dalila Berbagui, “Commerce et petite entreprise étrangère dans la ville (1980-2002)”, in Ethnologie française, vol. 35, $n^{\circ}$ 1, 2005, pp. 109-115. 10. Chantal Crenn, "Modes de consommation des ouvriers agricoles originaires du Maroc installés dans la région de Sainte-Foy-la-Grande en Libournais (Gironde)", in Anthropology of Food, octobre 2001.

11. Florence Bergeaud-Blackler, Les Marchés de viande halal en Aquitaine, Bordeaux, Aquibev- ministère de l'Agriculture (DGAL), 2001. 12. Plat dans lequel on prépare la semoule de couscous avant de la faire cuire à la vapeur. 
ou des boissons au gingembre qualifiées de "bières sans alcool”. De même, des saucissons de volaille à "farce claire" ou "farce sombre" côtoient, au rayon frais, les salamis de dinde au packaging quasi identique à celui des produits non halal qui leur correspondent.

D'autre part, ces commerces ethniques, implantés parfois au sein d'anciens quartiers à forte concentration d'immigrés et en cours de gentrification, se sont découvert une nouvelle clientèle pour laquelle ils vont également diversifier leur offre. Une clientèle "bourgeois-bohème", attirée par le caractère cosmopolite de ces quartiers $^{13}$, vient s'approvisionner dans ces commerces dans lesquels l'échange avec les clients est privilégié. On peut s'y procurer à des heures tardives des ingrédients orientaux et goûter les pâtisseries vendues souvent au prix fort. Un épicier casablancais de Nancy nous montre la grande variété des produits de sa boutique qui s'oriente de plus en plus vers des ingrédients spécifiques de la cuisine subsaharienne, indienne ou japonaise: "Il y a des personnes aux origines très diverses qui viennent ici, mais il y a aussi des Français qui veulent retrouver un ingrédient qu'ils ont goûté lors de leurs vacances à l'étranger." Cet entrepreneur qui part bientôt à la retraite diversifie son offre afin que le client trouve toujours l'ingrédient dont il a besoin, si rare soit-il : «Je dois pouvoir satisfaire le client afin qu'il ne soit pas obligé de parcourir toute la ville pour trouver l'ingrédient recherché. C'est comme cela qu'on fidélise les gens."

Cependant, cette diversification ne trouve pas ses racines uniquement dans l'évolution des goûts de la population. Les modes de consommation en sont aussi à l'origine. En effet, de la même façon que les commerces français de proximité ont été mis en danger par l'apparition de la grande distribution à partir des années 1960, ce dont les commerces ethniques étaient assez protégés jusqu'alors grâce justement à la spécificité de leurs produits, ceux-ci doivent faire face aujourd'hui à l'offre gran- dissante en denrées maghrébines et orientales des supermarchés qui, de plus en plus, disposent d'un rayon "cuisine du monde". Plus encore, le commerçant de proximité rencontre aujourd'hui la concurrence d'un nouveau circuit de distribution, celui des grandes surfaces spécialisées dans les produits maghrébins ou orientaux de manière générale, regroupant épicerie, boulangerie et boucherie. La chaîne "Saveurs d'Orient", implantée en Lorraine,

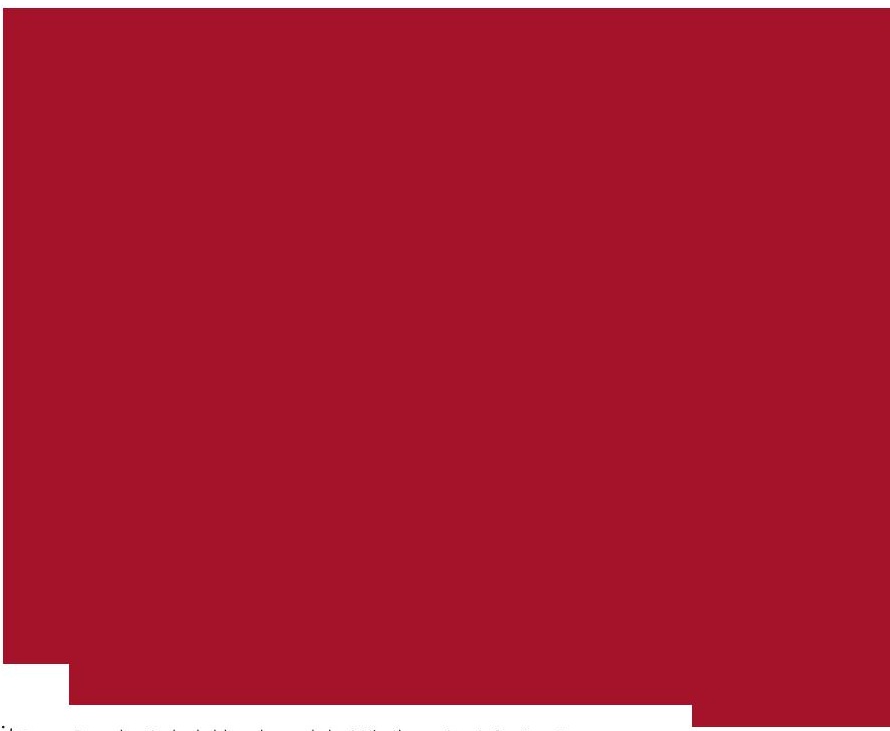

Boucherie halal boulevard de Ménilmontant, Paris $11^{\mathrm{e}}$ (c) MARIE CAQUeL

ouvre ses portes en 1996 ainsi qu'une première succursale l'année suivante dans un département voisin.

Enfin, la diversification de l'offre trouve ses raisons dans la saturation de certains quartiers en commerces ethniques. Certaines rues, telle celle de Ménilmontant à Paris, sont constituées d'une enfilade de commerces en alimentation : la tendance est donc à la diversification d'activités au sein même de la boutique. C'est pourquoi les boucheries halal ne proposent rarement que de la viande et font souvent office d'épicerie, ce qui semble porter ses fruits. Après trente ans d'expérience dans cette 
activité et l'ouverture de son commerce en 2007, ce boucher de Nancy aménage début 2013 son arrièreboutique en épicerie : "Souvent les clients paient par Carte bleue, ça les arrange de pouvoir payer tout sur place, la viande et son accompagnement. Au lieu d'aller à l'épicerie d'à côté, ils achètent tout chez moi !" Ainsi, parmi les artisans, commerçants et chefs d'entreprise marocains, au nombre de 10626 en France en $2009^{14}$, ceux qui ont investi dans le secteur de l'alimentation développent une activité qui non seulement s'adapte à l'évolution des pratiques alimentaires communautaires, mais s'ouvre aussi à une population de plus en plus curieuse et adepte de la cuisine "qui vient d'ailleurs". Cette activité, certains entrepreneurs veulent la faire fructifier pour accomplir un projet inscrit dans leur circuit migratoire : investir dans le pays d'origine.

\section{Le commerce et la restauration en pointe des investissements}

Dans un contexte général de mondialisation des mobilités humaines ${ }^{15}$, les tendances migratoires actuelles donnent lieu à l'émergence de nouvelles figures de migrants marocains.

Ces derniers deviennent des "circulants" et s'inscrivent, eux aussi, dans cette logique de pratique de l'espace, à cheval entre le pays de départ et celui d'installation. En s'appuyant sur des réseaux transnationaux, ils parcourent des territoires circulatoires $^{16}$ et développent des activités économiques, politiques et sociales au-delà des frontières. Ces migrants transnationaux disposent d'importants 
atouts qui ne tiennent pas seulement à leur nombre et à leurs apports économiques, mais aussi à leur attachement affectif au Maroc et à leurs compétences. Bon nombre d'entre eux investissent au Maroc tant leurs fonds épargnés à l'étranger que leur expérience acquise au cours de leur parcours migratoire.

L'immobilier constituait le secteur d'activité qui drainait l'essentiel de l'investissement des Marocains résidant à l'étranger. Il est le symbole d'une migration réussie et le signe d'un sentiment d'attachement au pays d'origine. Toutefois, le Maroc constitue aujourd'hui une destination pour une nouvelle catégorie d'entrepreneurs transnationaux qui porte de plus en plus d'intérêt aux secteurs économiques productifs, en l'occurrence le secteur tertiaire. Les entreprises créées sont souvent de petits projets. Ainsi, l'acquisition d'un petit commerce (épicerie, supérette), d'un café, d'un restaurant ou d'une boulangerie semble le plus adéquat pour ces migrants. Ce sont des investissements qui réalisent un bénéfice immédiat, ne nécessitent pas un grand capital financier et ne requièrent pas non plus un savoir-faire particulier.

D'après les résultats d'une enquête menée auprès de ces investisseurs à Agadirr ${ }^{17}$, deux catégories d'entrepreneurs transnationaux se dégagent. La première concerne des anciens émigrés partis dans les années 1970. Ils ont tous le même pays d'arrivée : la France. Pour certains, l'investissement à Agadir se situe dans le prolongement de leurs projets commerciaux créés antérieurement dans l'Hexagone. Avec un âge supérieur à celui de la retraite, ces investisseurs s'inscrivent dans un héritage culturel qui confirme la spécialisation des Soussis dans le commerce. Ces derniers pratiquent cette activité économique au départ comme à la fin de leur cycle migratoire. Le témoignage de "l'haj" Taîb va dans ce sens, il nous raconte son histoire de commerçant de l'entre-deux : "En 1975, mon père a décidé de me faire venir en France pour l'aider à gérer sa petite épicerie. Lorsque notre activité commerciale s'est développée, nous avons décidé d'acheter un autre maga-

sin plus grand à Gennevilliers. Vingt-cinq ans plus tard, j'ai décidé d'investir dans mon pays. J'ai opté pour le commerce car c'est le seul métier que je sais faire !" Succédant à son père, aujourd'hui l'“haj” Taîb détient une supérette moderne à Agadir et une autre similaire à Gennevilliers. Malgré son âge avancé, il se place dans un mouvement de va-et-vient entre le Maroc et Paris.

La seconde catégorie est composée de migrants âgés de 30 à 50 ans partis à l'étranger pour terminer leurs études universitaires ou à la recherche d'un avenir meilleur dans le cadre d'une migration de travail légale ou illégale. Ils étaient employés dans leur pays d'installation et sont devenus leur propre patron au Maroc. Plus instruits et dotés d'une formation professionnelle plus importante que la catégorie précédente, ils ont un autre comportement en matière d'investissement. Kamal, âgé de 40 ans, nous explique sa démarche entrepreneuriale : "Le Maroc a beaucoup changé. Aujourd'hui, il est plus avantageux d'investir ici qu'en Europe. L'étude

Taîb détient une supérette moderne à Agadir et une autre similaire à Gennevilliers. Malgré son âge avancé, il se place dans un mouvement de va-et-vient entre le Maroc et Paris. de marché que j'ai réalisée avant le lancement de mon restaurant a montré que ce projet dispose de toutes les chances pour réussir. Situé à proximité des facultés et connu par la qualité de ses plats et de son service, le restaurant est un espace moderne qui traduit mon parcours migratoire." En effet, pour répondre aux attentes de ses clients, en majorité des étudiants, Kamal propose une panoplie de menus diversifiés et à petits prix. Selon lui, les sandwichs, les hamburgers, les panini ou encore les pizzas, sont les mets les plus commandés par ces jeunes qui n'ont ni le temps ni l'envie de préparer à manger à domicile.

Kamal vit aujourd'hui entre les deux rives de la Méditerranée. Pour être avec ses enfants résidant en Belgique, il se déplace plusieurs fois par an entre Agadir et Anderlecht. Durant son absence, son frère s'occupe de la gestion du restaurant. 
L'investissement de ces migrants transnationaux au Maroc remet en cause les motivations de leur acte entrepreneurial ainsi que les circonstances dans lesquelles il s'est construit. À travers leurs projets, ces migrants recherchent une réinsertion autant économique que sociale dans leur pays d'origine. Une réinsertion qui reste, parfois, relativement difficile avec une identité multiple forgée au cours de l'expérience migratoire ${ }^{18}$.

\section{Manger japonais à Agadir}

La gastronomie est devenue un indicateur de mondialisation $^{19}$. Le secret de la réussite du secteur réside dans la juxtaposition d'un ensemble d'aspects touchant la qualité de l'offre, l'hygiène alimentaire, le marketing et l'évolution de la demande d'une clientèle qui souhaite voyager à travers son assiette. Par conséquent, le paysage gastronomique marocain s'est diversifié et les pratiques culinaires ont évolué. En effet, depuis son indépendance en 1956, le Maroc a connu de réelles mutations tant sur le plan démographique que social. Le développement de l'activité économique dans les centres urbains a participéa l'évolution des modes de consommation des citadins. Parmi eux, une nouvelle catégorie de Marocains recherche la diversification de ses habitudes alimentaires courantes. Si ceux-ci affectionnent les recettes traditionnelles revisitées et préparées autrement, ils découvrent aussi, attirés par les goûts venus d'ailleurs, une cuisine variée émanant des quatre coins du monde. Les citadins peuvent ainsi apprécier les mezze libanais, les plats indiens et même le foie gras français. En fréquentant les restaurants, ces Marocains visent aussi à affirmer et à afficher leur statut social aux yeux d'autrui. Dans le Maroc d'aujourd'hui, manger au restaurant est désormais un acte de modernité, mobilisé à des fins de distinction sociale ${ }^{20}$ dans la mesure où cette pratique n'est pas à la portée de tous.

Dans ces circonstances, la restauration est devenue un secteur florissant qui incite des migrants transnationaux à investir dans leur pays d'origine. Ces entrepreneurs agissent sur le contenu de l'offre gastronomique nationale et concourent au progrès de sa diversité en proposant de nouveaux mets à la consommation. Parmi eux, Saïd fait figure d'exemple. Après un long séjour en Allemagne où il a travaillé comme restaurateur, il ouvre un restaurant japonais dans le quartier touristique d'Agadir. Baptisé Saito-Kan - "le palais de Saïd" -, ce restaurant accueille chaque jour des Marocains, plus que des touristes, venus déguster les saveurs de la gastronomie japonaise. Préparés par deux chefs cuisiniers japonais installés à Agadir, les sushis restent le plat vedette commandé par les clients. Saïd nous confirme que "manger japonais est devenu à la mode dans les grandes villes marocaines" et que "seules quelques classes sociales peuvent y accéder. Celles-ci connaissent aujourd'hui les makis, les sashimis et les nigiris". Un an seulement après l'ouverture de son restaurant, Saïd pense joindre aux menus proposés à ses clients une nouvelle gamme de plats coréens. Les migrants marocains transnationaux d'aujourd'hui se trouvent entre deux sociétés en transformation constante en termes de pratiques sociales et culturelles. Entre mondialisation et affirmation identitaire, l'acte entrepreneurial en France comme au Maroc dans le secteur de la gastronomie devient un marqueur des évolutions des pratiques alimentaires de chaque côté de la Méditerranée. Les migrants marocains, à travers leurs stratégies entrepreneuriales, ont un statut hybride consistant à être garants des traditions marocaines en France tout en se faisant acteurs de mondialisation dans les deux pays. 